\title{
Transverse ion diffusion inside fixed charge narrow pores
}

\author{
B.Sulbarán ${ }^{1,2}$, W.Olivares-Rivas ${ }^{1}$, P.J.Colmenares ${ }^{1}$ \\ 1 Grupo de Química Teórica Quimicofísica de Fluidos y Fenómenos \\ Interfaciales (QUIFFIS), Departamento de Química, \\ Facultad de Ciencias, Universidad de Los Andes, Mérida, Venezuela \\ 2 Departamento de Investigación, Area de Ciencias Aplicadas, \\ Instituto Universitario Tecnológico de Ejido (IUTE), Ejido, Venezuela
}

Received November 9, 2004

The diffusion dynamics of ions inside a planar slit with a high constant surface charge density was studied by solving the Smoluchowski/HypernnetedChain equation for the mean first passage times. The behavior of local properties, such as the potential of mean force, is correlated with the time it takes for an ion to get adsorbed at the surface, and also with macroscopic properties like the salt rejection coefficient and the apparent diffusion constant in porous media, as a function of pore size. We show that the width of the walls has an important effect on the transverse ion diffusion process.

Key words: ion diffusion, charged micro-pores, mean first passage time, apparent transverse diffusion constant

PACS: $05.20 . J j, 82.65 .+r$

\section{Introduction}

The development of reliable techniques for analyzing and visualizing the solid surface in a liquid-solid interface, where catalytic reactions, ion exchange or adsorption might be occurring, has focused the attention into the surface processes, with great success. On the other hand, processes taking place in the liquid region are still being interpreted using classical theories, many times beyond their limit of validity. Most of the fundamental discussions or interpretations of experimental results on interfacial processes, such as ion diffusion and exchange, electrochemistry, surface chemistry and heterogeneous liquid-solid catalysis, are based on the a priori assumptions that ion concentration and charges are small, surface zeta potentials are constant and low, ions are point charges and that membranes, pores and its walls are infinitely wide. We have shown in the past that, when those restrictions are overcome, important nonlinear effects become significant [1-3].

The diffusion of ions in concentrated bulk solutions is by itself a difficult prob- 
lem, since long range electrostatic correlations are coupled with volume exclusion and other short range dispersion interactions, modulated by the polar solvent $[4,5]$. Inside a charged micro pore, the electrostatic field created by the charged walls sets up a non-homogeneous distribution of the mobile ions in solution. Therefore, the transverse diffusion (perpendicular to the surface) is further complicated by such non-homogeneous environment near the interface. The lateral diffusion (parallel to the interface) is affected to a lesser degree by the charged walls since the average lateral forces are null [6].

In a previous work [7], hereafter referred to as paper I, we discussed the ionmembrane interactions from a statistical mechanics point of view. The ion dynamics in the vicinity of an isolated charged membrane of finite width was described by solving the Smoluchowski equation for the Mean First Passage Time of co-ions and counter-ions, $\tau_{j}(x)$. That is, we calculated the mean time it takes for and ion at a given position $x$ away from a membrane to reach its absorbing surface. There we showed that the use of the classical theory (the point ion Modified Gouy-Chapman theory, MGC) for the density profiles leads to a monotonic behavior of $\tau_{j}(x)$ as a function of $x$, while the use of more accurate profiles, such as the integral equation HNC/MSA, gives rise to oscillations about the values of a free diffusing particle, particularly for high ionic and surface charges.

In this work we examine the diffusion dynamics of the ions of an aqueous solution enclosed in narrow slit pores bearing a fixed surface charge density $\sigma$ in the internal face of the walls. Particular attention will be given to the effect of the ion-ion correlation across pore walls of finite thickness on the diffusion process.

\section{Theory}

To simulate a slit pore we consider two planar slabs of width $d$, bearing a constant surface charge density $\sigma$ and separated by a distance $L$, immersed in a primitive model ionic solution of charged hard spheres of finite diameter $a$. The static equilibrium properties of this system has been solved by us under a variety of ion and surface charges, salt concentration, pore sizes and wall widths conditions $[1,2]$. Details of the model and theory used are given in $[2,3]$ and in paper I [7].

The ions are free to move in the $\mathrm{y}$ and $\mathrm{z}$ directions parallel to the walls, but are confined to the transverse diffusion $x$-domain. The most common model boundary condition is to fix the electrostatic potential or even the so-called zeta potential. However, most real systems have a fixed, self regulated and discrete surface charge which is well represented by a smeared surface charge density $\sigma$. Even though the surface charge densities in the inner wall faces, $\sigma_{\text {in }}$, and in the outer wall faces, $\sigma_{\text {out }}$, could have any arbitrary fixed value, we have chosen here to let $\sigma_{\text {in }}=\sigma$ and $\sigma_{\text {out }}=0$. In this way, the solution outside the pore is in front of a hard wall that has no interaction with the solution inside the pore. This will allow us to clearly observe the correlation between the solutions inside and outside the pore $[1,2]$.

The ions are assumed to move according to a Langevine-like dynamics [3]. In the presence of an electrical double layer, besides the hydrodynamic drag force and the 
white noise force resulting from the collisions of the particles with ion $j$, a net force $-\nabla W_{j}(x, t)$ also acts on the ions of species $j$, where $W_{j}(x, t)$ is the total potential of mean force. By definition, $-\beta W_{j}(x)=\ln \left[\rho_{j}(x) / \rho_{j}\right]$, where $\rho_{j}(x)$ is the local density profile inside the pore and $\rho_{j}$ is the ion density in the bulk electrolyte solution.

In the so-called Instantaneous Relaxation Approximation (IRA), the time dependent correlation effects are neglected under the assumption that, as a central ion moves inside a charged pore, the other confined ions adjust their spatial configuration instantaneously. That is to say, the electrical double layer relaxes on a much faster time scale than the diffusion process. The IRA is then equivalent to the assumption that a given central ion would diffuse against the barrier of the one-particle equilibrium potential of mean force set up by the charged walls, $W_{j}(x, t)=W_{j}(x)$. A direct molecular dynamics test of the goodness of this approximation was carried out by Jönsson et al $[6,8]$.

We shall focus our discussion on the determination and analysis of the mean time taken by a given ion $j$ to reach one of the walls, namely, the Mean First Passage Time $(\mathrm{MFPT}), t_{j}^{\mathrm{MFP}}(x)$. To be comprehensive, we briefly give here the basic concepts.

Let $P_{j}(y, t \mid x, 0)$ be the conditional probability of finding an ion $j$ in position $y$ at a time $\mathrm{t}$, given that it started to diffuse from a position $x$ at the initial time $t=0$. Under the IRA approximation, the backward Fokker-Plank equation can be written [9]

$$
\frac{\partial P_{j}(y, t \mid x, 0)}{\partial t}=-D_{j} \frac{\mathrm{d} \beta W_{j}(x)}{\mathrm{d} x} \frac{\partial P_{j}(y, t \mid x, 0)}{\partial y}+D_{j} \frac{\partial^{2} P_{j}(y, t \mid x, 0)}{\partial y^{2}},
$$

where $D_{j}$ is the bulk diffusion constant. The symmetry in the $x$-domain perpendicular to the surfaces ensures a reflecting plane located at $x=0$, a mid distance $h=(L-a) / 2$ from the plane of the closest approach to the pore walls.

The probability of an ion to be located at any point within the diffusion domain $[-h, h]$ without having reached the walls, given that it was at the initial position $x$ is the so-called survival probability $G_{j}(x, t)$ :

$$
G_{j}(x, t)=\int_{-h}^{h} P_{j}(y, t \mid x, 0) \mathrm{d} y .
$$

In terms of the survival probability, the probability density for the first passage times is $-\partial G_{j}(x, t) / \partial t$. The first moment of this distribution is the Mean First Passage Time, $t_{j}^{\mathrm{MFP}}(x)$

$$
t_{j}^{\mathrm{MFP}}(x)=-\int_{0}^{\infty} \frac{\partial G_{j}(x, t)}{\partial t} t \mathrm{~d} t=\int_{0}^{\infty} G_{j}(x, t) \mathrm{d} t .
$$

When equation (1) is integrated over the position and then over time, according to equations (2) and (3), we obtain, under the IRA approach, an ordinary differential equation which is satisfied by the MFPT, and referred to as the Smoluchowski equation:

$$
\frac{\mathrm{d}^{2} \tau_{j}(y)}{\mathrm{d} y^{2}}-\frac{\mathrm{d} \beta W_{j}(y)}{\mathrm{d} y} \frac{\mathrm{d} \tau_{j}(y)}{\mathrm{d} y}=-1
$$


where we have defined the dimensionless $\operatorname{MFPT} \tau_{j}(y)=D t_{j}^{\mathrm{MFP}} / h^{2}$, and have scaled the positions as $y=x / h$.

This equation can be solved analytically for several absorbing or reflecting mathematical boundary conditions [3,9-12]. A boundary is said to be absorbing if the diffusion process terminates when the particle reaches that boundary. Then at an absorbing surface $\tau_{j}(x)=0$. Physical adsorption, ion exchange or a chemical reaction would make a surface a mathematical absorbing boundary. The mathematical terminology reflecting boundary is used if, when the particle reaches a point on that plane, it is transferred to a point within the diffusing domain. In a pore with equal surface charge densities on the opposing walls, the symmetry mid-plane is a reflecting boundary by definition. At the mid-plane $x=0$ the mean first passage time must satisfy $\mathrm{d} \tau_{j}(x) / \mathrm{d} x=0$. Then, it would be equivalent to consider an entire diffusing domain between two absorbing surfaces at $x=-h$ and $x=h$, or half the domain between the reflecting midplane plane at $x=0$ and the absorbing plane at the plane of closest approach $x=h$.

For a symmetrical pore with absorbing walls we then have $[11,12]$

$$
\tau_{j}(x)=\int_{\frac{x}{h}}^{1} \frac{\mathrm{d} y}{\rho_{j}(y)} \int_{0}^{y} \rho_{j}(z) \mathrm{d} z
$$

In the absence of correlations, the potential of mean force vanishes, $W_{j}(y)=0$ and $\rho_{j}(y)=\rho_{j}$. Thus, from equation (5), we get the dimensionless free diffusing particle limit as $\tau_{j}^{0}(x)=\left(1-(x / h)^{2}\right) / 2$. From this result, the mean first passage time for diffusion from the midpoint of the pore at $x=0$ to a wall at $x=h$ is $t_{\mathrm{B}}=h^{2} / 2 D$, the well known one dimension brownian motion result.

The local ion concentrations change markedly in the neighborhood of a charged interface in order to adjust the chemical potential change under the effect of the electrostatic field and the ion-ion correlations. This is well described with the generalized expression for the chemical potential $\mu_{j}$ of ionic species as a local property in a non-homogeneous solution next to a charged surface [13].

$$
\beta \mu_{j}(r)=\beta \mu_{j}^{0}+\ln \rho_{j}(r)+\beta v_{j}(r)-C_{j}(r),
$$

where $\rho_{j}(r)$ is the local particle density profile for species $j$ at a point $r$ in space, $\beta=1 / k T, k$ is Boltzmann's constant, $T$ is the temperature, and $v_{j}(r)$ is the bare external electrostatic potential acting on the ions of species $j$ in solution, excerpted by the hard charged pore walls. In equation (6), $\mu_{j}^{0}$ is the chemical potential in the ideal gas reference state, $\beta \mu_{j}^{0}=\ln \Lambda_{j}^{3}, \Lambda_{j}=\hbar\left(m_{j} k T / 2 \pi\right)^{-1 / 2}, \hbar$ is Planck's constant, $m_{j}$ is the ion mass and $C_{j}(r)$ is the one particle correlation function.

At equilibrium, the local chemical potential $\mu_{j}(r)$ is a constant everywhere. The position dependent ionic profile or local density inside a micro-pore, $\rho_{j}(r)$, in equilibrium with a reservoir solution with bulk densities $\rho_{j}$, is expressed in the convenient form

$$
\rho_{j}(r)=\rho_{j} \mathrm{e}^{-\beta\left[e_{j} \psi(r)+J_{j}(r)\right]},
$$

where the potential terms collected in the exponent give the total potential of mean force, $\beta W_{j}(r)=-\ln \left(\rho_{j}(r) / \rho_{j}\right)$. The potential $J_{j}(r)$ is obtained by solving the exact 
relationship obtained by Lovett, Mou and Buff [14] and Wertheim [15], known as the LMBW equation,

$$
\nabla_{1} J_{j}\left(r_{1}\right)=-k T \sum_{i} \int \nabla_{2} \rho_{i}\left(r_{2}\right)\left(C_{i j}\left(r_{1}, r_{2}\right)+\beta \frac{e_{i} e_{j}}{\epsilon r_{12}}\right) \mathrm{d} \mathbf{r}_{2},
$$

where $C_{i j}\left(r_{1}, r_{2}\right)$ is the two particle direct correlation function.

The mean electrostatic potential $\psi(x)$ is obtained by integration of Poisson's equation. The equilibrium profile is given by the Boltzmann factor $\rho_{j} \mathrm{e}^{-\beta e_{j} \psi(x)}$ only when the short range correlation potential term $J_{j}(x)$ is neglected, giving the Poisson-Boltzmann (PB) or Gouy-Chapman (GC) theory. Inclusion of a plane of closest approach is referred to as the Modified Gouy-Chapman theory (MGC). The MGC theory takes into account the short range ion-interface repulsion, but neglects the ion-ion correlations and ionic size effects. To take these effects into account, one has to solve equations (8) and (7) for $\rho_{i}(r)$.

One can choose to solve the singlet LMBW equation, equations $(8)$ to $(7)[17,18]$ or, equivalently, the solution of the HNC/MSA singlet integral equation, $[16,19,20]$. The simplest anzatz for $C_{i j}\left(r_{1}, r_{2}\right)$ is to take it as the bulk two particle direct correlation function in the MSA approximation, which is known analytically. Such approximation corresponds to the singlet HNC/MSA approximation solved by LozadaCassou et al [16] for a number of geometries of different complex colloidal particles. Improvement can be obtained from Monte Carlo (MC) numerical simulations [21,24], functional density theory [25], or using a better approximation for $C_{i j}\left(r_{1}, r_{2}\right)$ in the LMBW equation [26].

Therefore, it is fare to say that at equilibrium the ion profiles near a planar interface, the mean electrostatic potential and therefore the total potential of mean force acting on the ions approaching the interface are well known.

\section{Results and discussion}

It is well established that the MGC theory is only valid for monovalent ions at low ionic strengths, below $0.01 \mathrm{M}$ and low surface charges, of the order of $500 \AA^{2}$ per site, which fortunately are the conditions of many important systems. However, for concentrated solutions and high surface areas, the HNC/MSA is in excellent agreement with the computer Monte Carlo and molecular dynamics simulations experiments [24], but important differences were found between the MGC and the formal theories [2].

As discussed in paper I for a single membrane, the mean potentials and particle density profiles strongly depend on the surface charge density. For charges as high as $66.67 \AA^{2}$ (corresponding to about $0.24 \mathrm{C} / \mathrm{M}^{2}$ ), as found in phospholipid membranes, a monotonic behavior was obtained with a moderate agreement of the MGC and HNC/MSA theories. But for extremely high charge densities as $29.45 \AA^{2}$ (corresponding to $0.544 \mathrm{C} / \mathrm{M}^{2}$ ) and a $2: 2$ electrolyte, a net charge inversion is obtained in the diffuse ionic double layer. We have used here the rather large charge of $0.544 \mathrm{C} / \mathrm{M}^{2}$ used in the physics literature to allow comparisons and to clearly 
illustrate the resulting nonlinear effects, with the understanding that this represents an extreme situation.

We have chosen to vary the inside surface charge density $\sigma$ and to let the outside wall of the pore to be a rigid uncharged surface. Changing the width $d$ increases the effective distances between the ions in the solution outside as well as the surface charge and ions inside. As $d$ goes from infinity to zero the ions outside see the external wall changing from a hard plane to a fully charged wall. The resulting non-uniform distribution of co-ions and counter-ions on the outside of the pore is viewed by the ions inside the pore as an effective charge, which decreases the field of $\sigma$ and attenuates the potential of mean force inside the pore as $d$ decreases. Since the outside charge is zero, the changes on the potential of mean force $W_{j}(x)$ inside the slit pore, as $d$ changes, are then clearly a consequence of the ion-ion correlations across the wall. Having a non-zero charge density in the outside walls would simply introduce additional double layer profile changes on both sides $[2,23]$.
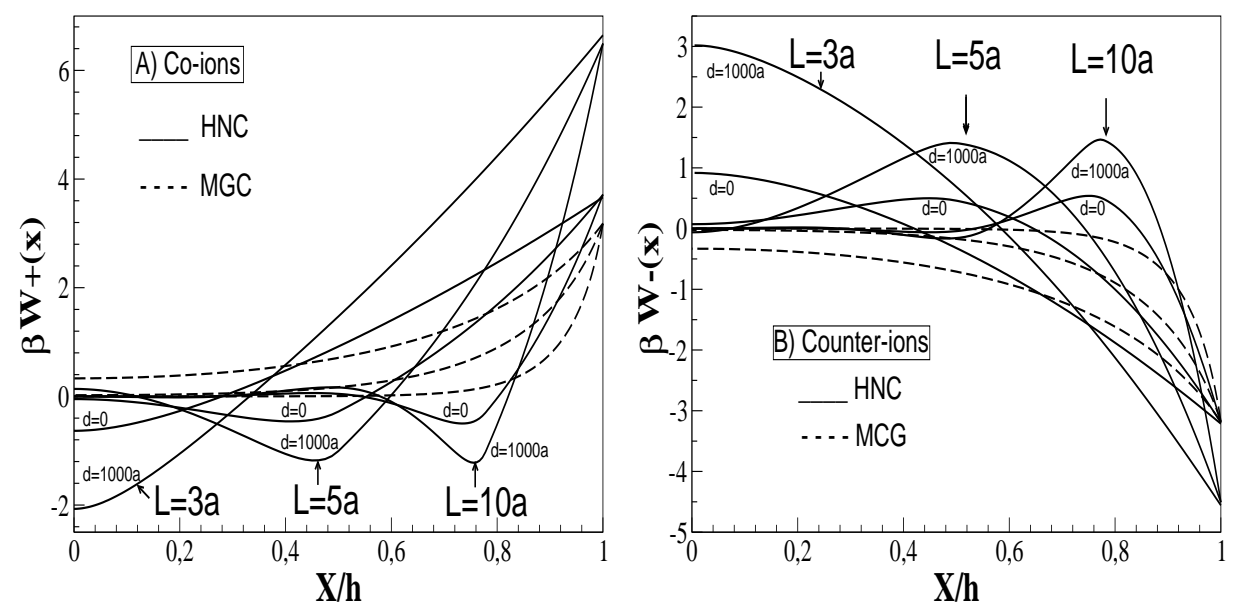

Figure 1. The reduced total potential of mean force $\beta W_{i}(x)$ acting on an ion $i$ as a function of the reduced distance $x / h$ to the pore midplane: A) co-ion, B) counterion. The system represents a primitive model of a planar pore, for $e / \sigma=66.67 \AA^{2}$ and $\rho_{b}=0.971 \mathrm{M}$ of a $2-2$ electrolyte at $298 \mathrm{~K}$ and $\varepsilon=78.5$. The dashed curves are the MGC results for $d=a$. The solid curves are the HNC/MSA singlet results for several values of the pore walls width $d$, as indicated. The surface charge on the outside wall face, $\sigma_{\text {out }}$ was set equal to zero.

The one membrane system studied in paper I [7] is equivalent to consider an infinitely wide pore with thin walls. Here we show the results for finite planar slit pores with wall to wall $L$ distances of the order of a few ionic diameters. In figure $1 \mathrm{~A}$ we show the reduced potential of mean force profile $\beta W_{+}(x)$ for doubly charged coions, for several values of the slit pore size $L, 10,5$ and 3 ionic diameters $a$ and wall width $d$ of 0 and 1000 diameters, as indicated. The RPM solution has $\rho_{b}=0.971 \mathrm{M}$ of a $2-2$ electrolyte at $298 \mathrm{~K}$ and $\varepsilon=78.5$. Here $x=0$ is at the mid-plane of the pore and $x=h$ is located at the inner plane of the closest approach to the walls.

$\beta W_{+}(x)$ represents the potential barrier where the ions diffuse. In figure $1 \mathrm{~A}$, the 
dashed lines show the typically repulsive potential predicted by the MGC approximation for walls with $d=a$. Though not shown in the figure, changing the value of $d$ produces only minor changes on the MGC curves. The solid lines show the HNC/MSA theory predictions, which includes the entropy effects due to volume exclusion and ion-ion correlations.

It can be seen in figure $1 \mathrm{~A}$ that, while close to the positive wall the positive co-ions feel the strong wall repulsion, there are regions within the pore where the positive ions are attracted by the positive wall. So, as a co-ion diffuses towards the surface, it sees an effective potential well. For very large pores this potential oscillates from repulsive to attractive. But, as the pore becomes very narrow, the interaction with both walls shifts the minimum of the well towards the middle of the pore. As the pore walls become thinner the potential wells become less pronounced.

Changing the width $d$ increases the effective distances between the ions in the solution inside the pore and the surface charge and ions on the outside. Since the outside surface of the pore in figure $1 \mathrm{~A}$ is a rigid uncharged surface, the changes on the potential profiles on the inner side are a consequence of the ion-ion correlations across the walls. The non-uniform distribution of co-ions and counter-ions on the outer solution is viewed by the ions on inner solution as an effective diffuse charge, which adds up to $\sigma_{\text {in }}$, increasing the mean electrostatic potential $\psi(x)$ as $d$ decreases and, therefore, increasing the total potential of mean force $W_{j}(x)$.

This effect of ion-ion correlation across the walls was first discussed by us when studying a solution confined in a slit pore of size L separated from infinitely large pores on both sides by walls of width $d$, bearing the same fixed charge $\sigma=\sigma_{\text {in }}=\sigma_{\text {out }}$ $[1,2]$. We observed that, for thin walls, breaking the symmetry of the system by decreasing the pore size and therefore changing the ions environment inside the pore, produced important changes on the particle density and potential profiles on the solution outside the pore. In order to keep the chemical potential constant, the ions compensate the symmetry breakage with the breakage of the local electroneutrality. The effect is enhanced by the presence of high charge asymmetric electrolites [22]. This process had long been recognized as the co-ion exclusion or salt rejection. One can also break the symmetry of a wall by having different surface charges on both sides. This case was studied with the same computer code by our collaborators [23]. The effect has been further studied and confirmed by Monte Carlo simulations [24].

In figure $1 \mathrm{~B}$ we show the reduced potential of mean force $\beta W_{-}(x)$ profile for the negative counter-ions for the same system of figure 1A. The MGC approximation (dashed lines) gives an attractive potential everywhere, which is simply the negative of the co-ion potential plotted in figure 1A. On the other hand, the HNC/MSA predicts a large attraction very close to the plane of the closest approach, but, for this high $\sigma$ repulsive potential barrier is created at lower distances.

In figure $2 \mathrm{a}$, we show the mean first passage time $\tau_{+}(x)$ of a co-ion as a function of the initial reduced position $x / h$ for different pore sizes $L$ and very wide walls $d=1000 a$. The plotted values correspond to the ratio $\tau_{+}(x) / \tau^{0}(x)$ where $\tau^{0}(x)$ is the limiting mean first passage time of a free diffusing particle with no interactions for the reflecting/absorbing boundary condition used here, $\tau^{0}(x)=\left(1-(x / h)^{2}\right) / 2$. 

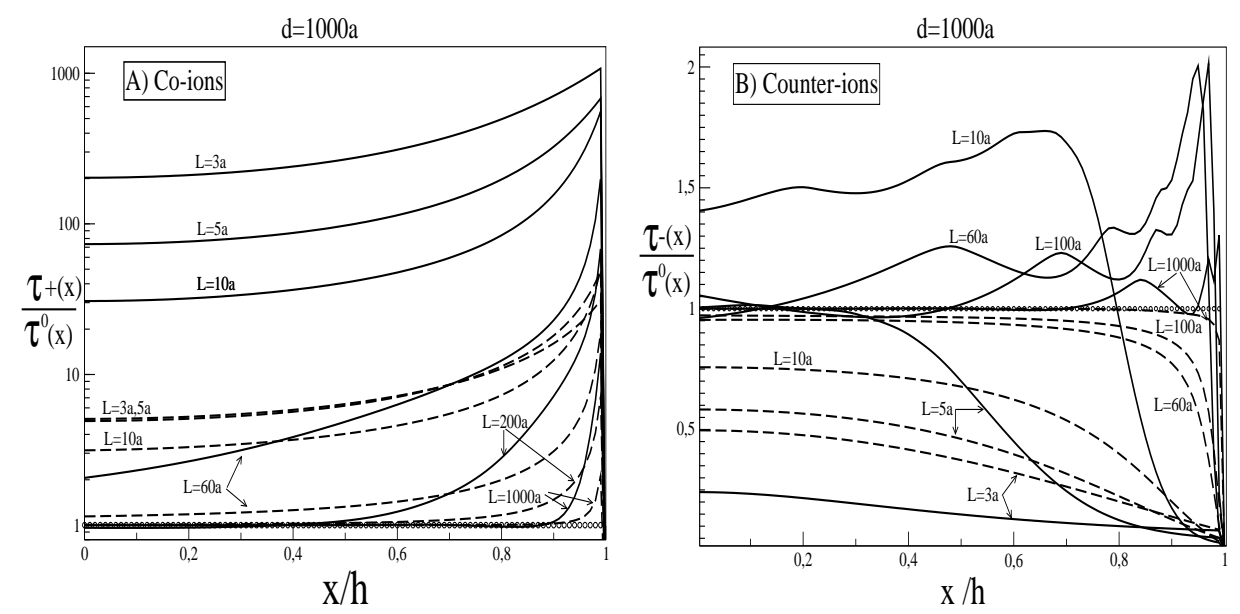

Figure 2. Ratio of the Mean First Passage Time of ions $j$ in a pore to the limiting MFPT of a free diffusing particle with no interactions, $\tau_{j}(x) / \tau^{0}(x)$, as a function of the initial position $x / h$, for the same system described in figure 1: A) co-ions; B) counter-ion. Solid lines corresponds to the S-HNC theory and the dashed line to points ions S-MGC approximation. Results are for very thick walls with $d=1000 a$ and pore sizes $L$ as labeled.

For very large pores we get the one membrane slab behavior reported in paper I [7], being indistinguishable from the non-interacting free diffusing particle for distances away from the walls. As the pore becomes smaller the behavior changes rapidly to a situation where $\tau_{+}(x)$ is independent of the initial position except for distances very close to the absorbing plane of the closest approach where it drops rapidly to zero. We can clearly see from the figure that the time it takes for a coion to get absorbed increases the free diffusing particle value $\tau^{0}(x)$ up to several times, as the pore becomes smaller. Thus for narrow pores the co-ions get trapped in the potential well described in figure 1A. The point ion Smoluchowski equation with the MGC profile S-MGC, shown in dashed lines, predicts times that are always monotonic and slightly larger than the free diffusing particle.

Figure $2 \mathrm{~b}$ shows the results for the counter-ion ratio $\tau_{-}(x) / \tau^{0}(x)$. Again, for very large pores, say for $L=100 a-1000 a$, we get the one membrane slab behavior reported in paper I [7]. That is, the MFPT ratio presents oscillations about $\tau_{-}(x) / \tau^{0}(x)=1$, for initial distances in the neighborhood of the interface. The mean first passage time ratio predicted by the HNC/MSA theory (continuos lines) changes in a non-monotonic way as a function of the initial position. As the pore becomes narrower, it first increases and then decreases. In figure 2B, the MGC (dashed lines) only accounts for the electrostatic attraction of counter-ions to the charged wall. As the pore becomes smaller, the time it takes to reach the surface decreases monotonically with the distance it starts from and decreases with respect to the free diffusing particle.

In figure 3 we show the effect of the ion-ion correlations across the walls on the Mean First Passage Time of ions $j$ in a pore of size $L=5 a$. We plot the deviation 

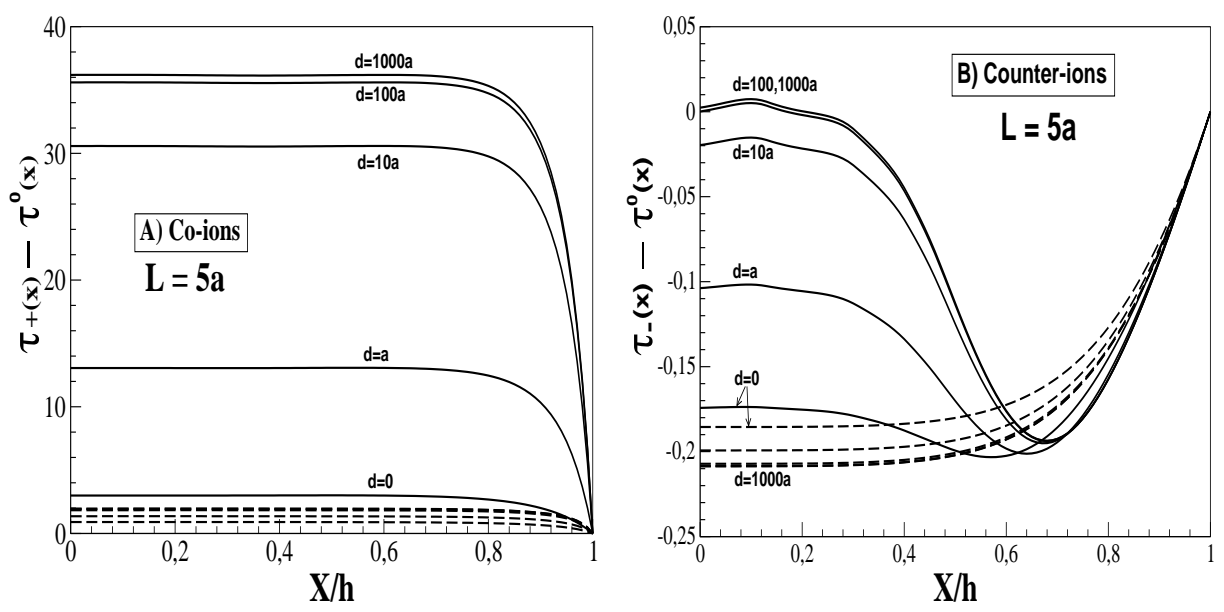

Figure 3. The effect of the ion-ion correlations across the walls on the Mean First Passage Time of ions $j$ in a pore of size $L=5 a$. We show the deviation of the MFPT of co-ions (A) and counter-ion (B) from the limiting MFPT of a free diffusing particle with no interactions, $\tau_{j}(x)-\tau^{0}(x)$, as a function of the initial position $x / h$, for several values of the wall thickness $d$ as indicated. The system and labels are the same as in figures 1 and 2 .

of the MFPT of co-ions (A) and counter-ion (B) from the limiting MFPT of a free diffusing particle with no interactions, $\tau_{j}(x)-\tau^{0}(x)$, as a function of the initial position $x / h$, for several values of the wall thickness $d$ as indicated. The system and labels are the same as in figures 1 and 2. For this pore size the electrostatic repulsion dominates and co-ions take longer than a non interacting particle to reach the absorbing boundary. For infinite walls no correlation exists among ions inside and outside the pore. As shown in figure 3A, for finite thickness the ion correlation gives rise to an effectively lower surface charge and the HNC/MSA MFPT decreases as $d$ decreases. The MGC approximation shows very little variation with $d$.

It is illustrative to analyze the behavior shown in figure 3 in the context of the mean force potential profile of figure 1 . For $L=5 a$ in figure 1 , the size or volume exclusion effect excludes counter-ions from the region around $x / h=0.5$, where coions form a layer. As the initial position moves away from the wall, the time shift for counter-ions, $\tau_{j}(x)-\tau^{0}(x)$ in figure $3 \mathrm{~B}$, goes through a minimum and then an inflection point. The counter-ions tend to have MFPT that are lower than the times for free diffusing particles since they are attracted to the wall at $x=h$.

In figure $3 \mathrm{~B}$ we can see that for distances to the walls lower than one diameter $(x / h>3 / 4$, with $h=2 a)$ the electrostatic attraction dominates and the counterion diffuse faster than a free diffusing particle showing little dependence on $d$. As the counter-ions start diffusing closer to the midplane the MFPT goes through a minimum and then increases, showing a clear dependence on the wall thickness. The point ion MGC approximation is monotonic and presents a small dependence on $d$ which goes in the opposite direction as that shown by the HNC theory.

Even though local properties, such as the local density profiles $\rho_{j}(x)$ or the MFPT 
$t_{j}^{\mathrm{MFP}}(x)$, are useful for understanding the physics occurring inside a micro-pore, most experimental measurements correspond to macroscopic quantities obtained from their space average. Moreover, in porous materials the measured quantities are the result of averages over a given array of micro-pores. Therefore we shall end this discussion by looking at such macroscopic quantities.

The average density of ions $j$ inside a pore is

$$
\bar{\rho}_{j}=\frac{1}{h} \int_{0}^{h} \rho_{j}(x) \mathrm{d} x .
$$

So, the normalized probability of finding an ion of species $\mathrm{j}$ at the initial position $x$ inside a pore is $g_{j}(x)=\rho_{j}(x) / \bar{\rho}_{j}$. The position average time that an ion $j$ takes to diffuse from any point inside the pore to the absorbing wall is then,

$$
\overline{t_{j}^{\mathrm{MFP}}}=\frac{1}{h} \int_{0}^{h} t_{j}^{\mathrm{MFP}}(x) g_{j}(x) \mathrm{d} x .
$$
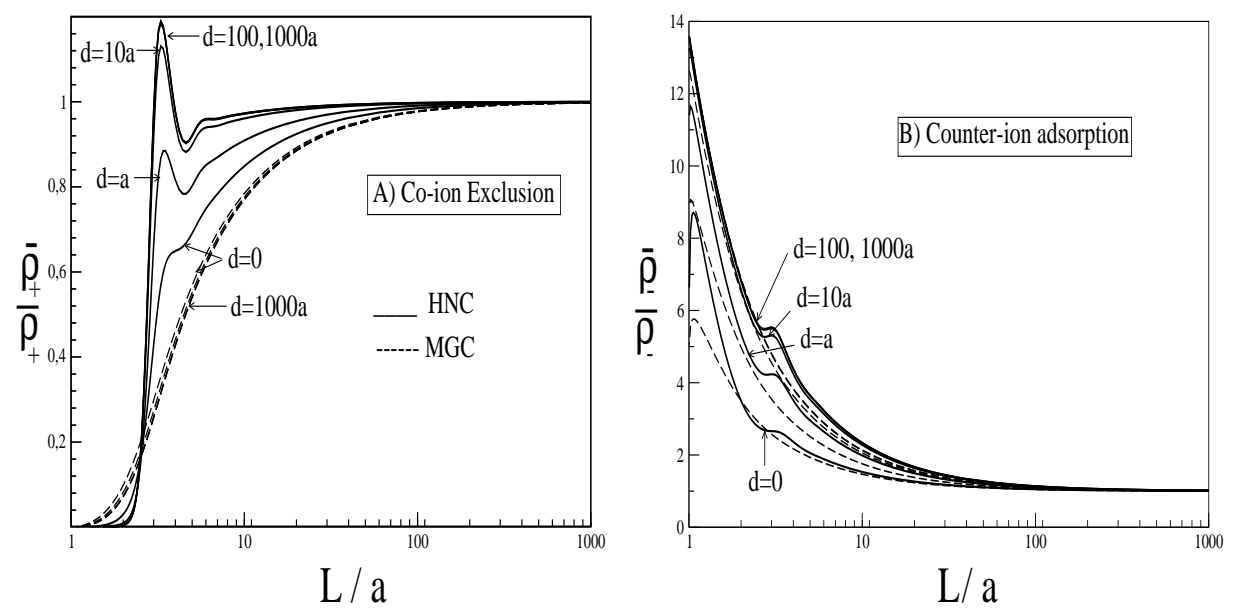

Figure 4. The local particle density,averaged over the entire pore, $\bar{\rho}_{j}$, as a function of the pore size $L / a$. Results are given for different wall thickness as labeled. A) $\bar{\rho}_{+} / \rho_{+}$shows the co-ion exclusion; B) $\bar{\rho}_{-} / \rho_{-}$shows the counter-ion adsorption.

For a free diffusing ideal particle confined in a pore of size $2 h, \rho_{j}(x)=\rho_{j}$, hence $\underline{g_{\circ}}(x)=1$ and $t_{\circ}^{\mathrm{MFP}}(x)=h^{2} /(2 D) \cdot\left(1-(x / h)^{2}\right)$. One gets ${\overline{t^{0}}}_{\mathrm{MFP}}=h^{2} /(3 D)$, or $\overline{\tau^{0}}=1 / 3$. By analogy, one can define an apparent diffusion constant $D_{j}^{a}$,

$$
\overline{t_{j}^{\mathrm{MFP}}}=\frac{h^{2}}{3 D_{j}^{a}} .
$$

In figure 4 we show the average density of co-ions and counter-ions, $\bar{\rho}_{j} / \rho_{j}$, inside a highly charge pore. $\bar{\rho}_{+} / \rho_{+}$is a measure of the so-called ionic exclusion or salt rejection in porous media. As shown by the MGC dashed lines in figure 1A, purely electrostatic effects give a monotonically increasing salt rejection, as the pore size 
decreases, with very little effect of the wall width $d$. That would also be the behavior of the HNC theory for low salt concentrations and low electrolyte and surface charges. A similar behavior is found when the surface or the zeta potentials are kept fixed as the pore size $h$ changes. However, for the high values of the fixed surface charge density $\sigma$ used here, the ion-ion correlations account for a local violation of the electroneutrality, which can be interpreted as a net surface charge reversal or overcharging [2]. This is reflected in the non-monotonic behavior of the co-ion exclusion, with a strong dependance on the wall width. Since the corresponding counter-ion concentrations are much higher this is seen in figure $4 \mathrm{~b}$ as a small hump in the adsorption isotherm $\bar{\rho}_{-} / \rho_{-}$vs pore size.
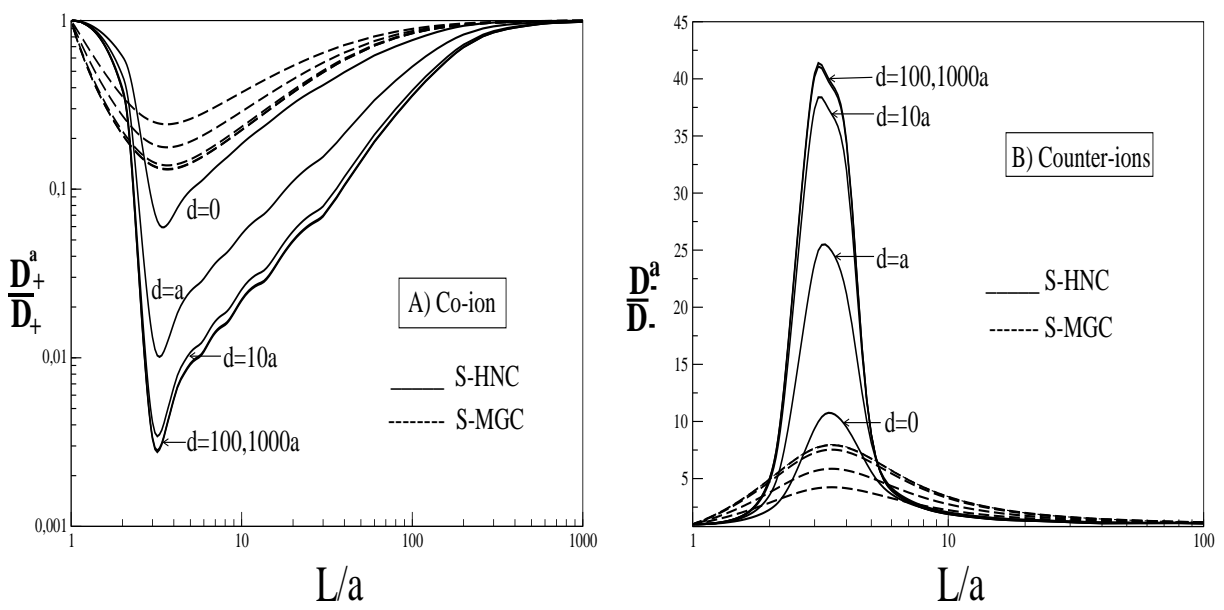

Figure 5. Ratio of the apparent transverse ion diffusion constant of confined ions to that of ions in the bulk solution, $D_{j}^{a} / D_{j}$, as a function of the pore size $L / a$, for different wall width $d$ as labeled. As before, solid lines correspond to the S-HNC approach, while the dotted lines correspond to the point ion limiting approach S-MGC.

Figure 5 shows the ratio of the apparent transverse diffusion constant $D_{j}^{a}$ of ions confined in porous media to the regular bulk solution ion diffusion constant $D_{j}$. As expected the ions diffuse to the absorbing charged walls as if counter-ions had a larger apparent diffusion constant $D_{-}$, and co-ions had a lower $D_{+}$. However, the transverse diffusion constants go through maxima and minima as the pore size becomes of molecular dimensions. The change in the drift of the ions in a porous media is much larger in the Smoluchowski-HNC description than in the Smoluchowski-MGC, showing the importance of the entropy size effects on the diffusion in confined systems. Figure 5B clearly shows the importance of ion-ion correlation across finite wall in reducing the enhancement of the diffusivity of counter-ions. For co-ions the thinner are the pore walls the higher is the apparent diffusivity, as shown in figure 5A. 


\section{Conclusions}

We have studied the transverse diffusion process of ions confined in a fixed surface charge planar micro-pore of several ionic diameters wide. We solved the FokkerPlank-Smoluchowski equation for the survival probability in terms of the mean Mean First Passage time for ions to reach the pore walls. The assumption of the IRA approximation allowed us the use of the MGC and HNC/MSA equilibrium potential profiles. The observed oscillations in the HNC particle density and the potential of mean force profiles in the vicinity of the pore walls gives rise to a similar behavior for the Smoluchoski-HNC (S-HNC) mean first passage time, $\tau_{j}(x)$, which oscillates about the value for a free diffusing ideal particle.

We also showed that the ion diffusion towards the interface is modulated by the ion-ion correlation across a thin pore wall. The results depend not only on the magnitude of the surface charge density at the inner and outer faces but also on the difference between them. For the conditions chosen, namely large inner surface charge and zero outer surface charge density, the effect of decreasing the pore wall width is to decrease the net charge density seen by the ions inside the pore. As a result, a compensation of the typical differences between the S-MGC and S-HNC theories for thin walls was observed for low values of $d$.

An apparent diffusion constant $D_{j}^{a}$ for a porous media was obtained and shown to depend strongly on size and ion-ion correlations within the pore and across the pore walls. The presented results should be useful in the interpretation of diffusion controlled processes occurring at highly charged interfaces. The classical MGC theory fails to show these features. In fact, it is useful only for very low surface charges and low concentrations of 1:1 electrolytes.

\section{Acknowledgement}

This work was supported by grant G97000741 from the Consejo Nacional de Investigaciones Científicas y Tecnológicas (CONICIT) of Venezuela and grant ADGCDCHT. We thank the Computer Center CeCalCULA.

\section{References}

1. Sulbarán B. Confined Electrolytes at Fixed Surface Charge: Electroneutrality and Static and Dynamic Properties. Thesis, Universidad de Los Andes, Mérida, Venezuela, 1996.

2. Lozada-Cassou M., Olivares-Rivas W., Sulbarán B., Phys Rev. E, 1996, 53(1).

3. Colmenares P.J., Olivares-Rivas W., Phys. Rev. E., 1999, 59, 841-849.

4. Wei D., Patey G.N., Torrie G.M., J. Phys. Chem., 1990, 94, 4260-4268.

5. Boda D., Henderson D., Patrykiejew A., Sokolowski S., J. Chem. Phys., 2000, 113(2), 802-806.

6. Jönsson B., Wenneström. Micellar Solutions and Microemulsions, chapt. 3, eds. S.H. Chen, R. Rajagopalan. Springer-Verlag, New York, 1990. 
7. Olivares-Rivas W., Fillous L., Sulbarán B. Ion-membrane Interactions. Encyclopedia of Surface and Colloid Science, p. 2892-2904, ed. A. Hubbard. Marcel Dekker Inc., New York, 2002.

8. Åkesson T., Jönsson B., J. Phys. Chem., 1985, 89(11), 2401-2405.

9. Gardiner C.W. Handbook of Stochastic Methods for Physics, Chemistry and the Natural Sciences. Springer-Verlag, Berlin, 1985.

10. Chan D.Y.C., Halle B., Biophys. J., 1984, 46(3), 387-407.

11. Chan D.Y.C., Chem. Soc. Faraday Trans. II, 1987, 83, 2271-2286.

12. Chan D.Y.C., McQuarrie D., J. Chem. Soc. Faraday Trans. II, 1990, 86(21), 35853595.

13. Evans R., Adv. Phys., 1979, 28, 143-200.

14. Lovett R., Mou C.Y., Buff F.P., J. Chem. Phys., 1976, 65(2), 570-572.

15. Wertheim M.S., J. Chem. Phys., 1976, 65(6), 2377-2381.

16. Lozada-Cassou M., Díaz-Herrera E., J. Chem. Phys., 1990, 92(2), 1194-1210.

17. Colmenares P.J., Olivares-Rivas W., J. Phys. Chem., 1986, 90, 1977-1979.

18. Colmenares P.J., Olivares-Rivas W., J. Chem. Phys., 1988, 88(5), 3221-3226.

19. Lozada-Cassou M., Henderson D.J. Statistical Mechanics of Interacting Double Layers. Micellar Solutions and Microemulsions, eds. Chen S.H., Rajagopalan R., p. 29-49. Springer-Verlag, New York, 1990.

20. Lozada-Cassou M. Fluid between walls and in pores. Fundamentals of inhomogeneous Fluids, ed. Henderson D.J., p. 303-361. Marcel Dekker, Inc., 1992.

21. Torrie G.M., Valleau J.P., J. Phys. Chem., 1982, 86, 3251-3257.

22. Lozada-Cassou M., Olivares W., Sulbarán B., Yu J., Physica A, 1996, 231, 197.

23. Lozada-Cassou M., Yu J., Phys. Rev. Lett., 1996, 77, 4019.

24. Degréve L., Lozada-Cassou M., Phys. Rev. E, 1998, 57, 2978-2983.

25. Henderson D., Bryk P., Sokolowski S., Wasan D.T., Phys. Rev. E, 2000, 61(4), 38963903.

26. Henderson D. Fundamentals of inhomogeneous Fluids, p. 303-361. Marcel Dekker, Inc., New York, 1992. 


\title{
Поперечна іонна дифузія всередині вузької пори з
} фіксованим зарядом

\author{
Б.Сулбаран ${ }^{1,2}$, У.Оліварез-Ріваз ${ }^{1}$, П.Дж.Колменарез ${ }^{1}$ \\ 1 Природничий факультет університету де Лос Андес, Венесуела \\ 2 Технологічний університет, Ексідо, Венесуела
}

Отримано 9 листопада 2004 р.

Дифузійна динаміка іонів всередині плоскої щілини з великою постійною густиною поверхневого заряду досліджувалась шляхом розв'язку супер-ланцюжкового рівняння Смолуховського для середніх часів перших переходів. Поведінка локальних властивостей, таких як потенціал середньої сили, корелює з часом, необхідним для іону бути адсорбованим на поверхні, а також з макроскопічними властивостями, такими як коефіціент несприйняття солі та константою уявної дифузії в пористих середовищах як функція розміру пори. Ми показуємо, що ширина стінок має суттєвий вплив на поперечний іонний дифузійний процес.

Ключові слова: іонна дифузія, заряджені мікропори, середній час перших переходів, константа уявної трансверсійної дифузії

PACS: 05.20.Jj, 82.65.+r 